\title{
Strategic gaze: an interactive eye-tracking study
}

\author{
J. Hausfeld ${ }^{1,2,3} \cdot$ K. von Hesler ${ }^{1,2} \cdot$ S. Goldlücke ${ }^{1}$
}

Received: 21 September 2018 / Revised: 31 March 2020 / Accepted: 6 April 2020 / Published online: 4 May 2020 (c) The Author(s) 2020

\begin{abstract}
We present an interactive eye-tracking study that explores the strategic use of gaze. We analyze gaze behavior in an experiment with four simple games. The game can either be a competitive (hide \& seek) game in which players want to be unpredictable, or a game of common interest in which players want to be predictable. Gaze is transmitted either in real time to another subject, or it is not transmitted and therefore non-strategic. We find that subjects are able to interpret non-strategic gaze, obtaining substantially higher payoffs than subjects who do not see gaze. If gaze is transmitted in real time, gaze becomes more informative in the common interest games and players predominantly succeed to coordinate on efficient outcomes. In contrast, gaze becomes less informative in the competitive game.
\end{abstract}

Keywords Eye-tracking $\cdot$ Focal points $\cdot$ Signaling $\cdot$ Hide \& seek

JEL Classification C91 $\cdot$ D01 $\cdot$ D83 $\cdot$ D87 $\cdot$ C72

\begin{abstract}
We thank Gary Charness, Sebastian Fehrler, Urs Fischbacher, Wolfgang Gaissmaier, Thomas Hattenbach, P.J. Healy, Ian Krajbich, Irenaeus Wolff, three anonymous referees, the editors and many participants at conferences and seminars for helpful comments and suggestions. This research has been financially supported by the Deutsche Forschungsgemeinschaft (DFG) through the Graduate School of Decision Sciences, the Research Unit Psychoeconomics, and Germany's Excellence Strategy.
\end{abstract}

Electronic supplementary material The online version of this article (https://doi.org/10.1007/s 1068 3-020-09655-x) contains supplementary material, which is available to authorized users.

J. Hausfeld

jan.hausfeld@uni-konstanz.de

1 Department of Economics, University of Konstanz, Konstanz, Germany

2 TWI Kreuzlingen, Kreuzlingen, Switzerland

3 Institute of Psychology, Social Neuroscience and Social Psychology, University of Bern, Bern, Switzerland 


\section{Introduction}

In 1960, Nobel laureate Thomas Schelling described a hypothetical experimental setting to test his theories as follows: "The first stage in the experiment is to invent a machine, perhaps on the principle of the lie detector, that will record or measure a person's recognition or the focus of his attention [...]" (Schelling 1960: 109). Since then, eye-tracking has developed into an affordable and precise method that is increasingly used in industry and research (Duchowski 2007; Holmqvist et al. 2011). Recently, it has attracted the attention of firms that develop technology for the twenty-first century such as eye controls for mobile devices, computer games, or smart homes. ${ }^{1}$ In research, eye-tracking has become a popular tool for the analysis of decision-making processes. In particular, it has been proven to be useful when studying how people play games and to infer players' levels of sophistication from recorded gaze data (Knoepfle et al. 2009; Wang et al. 2010; Stewart et al. 2016; Polonio et al. 2015). ${ }^{2}$ In contrast to this literature, we extend the use of eye-tracking technology to be part of the strategic interaction.

For this purpose, we use eye trackers connected to the software z-tree (Fischbacher 2007) such that players can see a real-time representation of the other player's gaze before making a choice. This setting is very close to what Schelling envisioned, and it opens up new possibilities for studying strategic interactions with gaze. If Schelling's prediction that each player "knows that his own visible reaction is yielding information about his own expectations" (Schelling 1960: 110) is true, then gaze that is transmitted to the other player should differ in predictable ways from gaze that is not transmitted. The aim of our study is to analyze how gaze and the interpretation of gaze change with the strategic environment.

Since eyes are a very natural means of communication, observing a person's gaze could help to coordinate if people share a common interest. There is ample evidence that individuals can learn from observing gaze in collaborative problem-solving tasks. $^{3}$ At the same time, the beneficial effects of gaze transfer are limited: Gaze cannot transmit complex information, and its dual use for visual search and for indicating a location creates ambiguities in the meaning of transmitted gaze. ${ }^{4}$

\footnotetext{
1 For example, Oculus bought The EyeTribe in 2016, Google bought Eyefluence in 2016, Apple bought SMI in 2017. Already, Eyeware raised 1.9 mio CHF and aims to develop technology that allows to use gaze to control gadgets in the apartment.

${ }^{2}$ Other studies use mouse-tracking for similar purposes (Costa-Gomes et al. 2001; Brocas et al. 2014). In comparison to mouse-tracking, eye-tracking is thought to be less intrusive in the decision making process as quick comparisons and information search are less affected by the recording mechanism (see Glöckner and Herbold (2011) and the literature cited therein).

3 See for example, Velichkovsky (1995), Stein and Brennan (2004), Litchfield et al. (2010), Litchfield and Ball (2011), Brennan et al. (2008), Neider et al. (2010). It has further been shown that people are generally better at interpreting dynamic rather than static representations of gaze in various types of tasks (Nalanagula et al. 2006; Gallagher-Mitchell et al. 2018; van Wermeskerken et al. 2018). While these studies analyze how the benefits of gaze transfer depend on the nature of the task environment, they do not consider the effects of varying incentives.

4 See e.g. Müller et al. (2013); Müller et al. (2014). The limits of eye-to-eye communication are nicely expressed in the science fiction novel "The Dark Forest", in which two of the characters try to convey a message through facial expressions: "They stared at each other, but held that pose for less than half a
} 
Observing gaze might not be helpful at all, if players have an incentive to be unpredictable or even to deceive another player. The question is how difficult people find it to keep their gaze from revealing their intentions in such situations. It could be that "eyes don't lie" and that gaze always conveys information even if this is not in the players' interest. ${ }^{5}$ For sender-receiver games without gaze transmission, Wang et al. (2010) show that there is substantial information about the intentions contained in eye movements and pupil dilation of the senders, potentially helping receivers to obtain a larger payoff at the expense of senders. In a recent study that compares nonstrategic gaze with deceptive gaze (Foulsham and Lock 2015), subjects are a lot less successful in guessing the choice made by a previously eye-tracked subject when the eye-tracked subjects are instructed to hide their true preferences. Success rates in the deceptive treatment are lower than in a baseline without any gaze transfer, butalthough guessers are not aware of the instructions-not lower than chance. In other contexts, the sincerity of eye movements is an illusion that might be exploited by liars. For example, many people, even professional interrogators, mistakenly believe that deception is associated with evasive eye movements (Vrij 2004).

To systematically test how people adapt their gaze when their focus of attention is shown to another player on screen, i.e. strategic gaze, we let subjects play four simple two-player games. We use three common interest games, in which being predictable is in the interest of the eye-tracked player, and a competitive hide \& seek game, in which being predictable can be exploited by the other player. In order to investigate differences between strategic and non-strategic gaze, we use a treatment in which gaze is recorded but not transmitted to the other player as a benchmark. This non-strategic gaze is later shown to participants in another treatment who then play against the respective gaze and choices. With this design, we test the limits of the ability to infer choices from gaze along two dimensions, varying the incentive to affect gaze (recorded gaze vs. live gaze transmission) and as well as the nature of the strategic interaction between the games (common interest vs. competitive).

We find strong evidence that people are able to correctly interpret non-strategic gaze patterns, both in the common interest games and also, but somewhat less successfully, in the hide \& seek game. When gaze is transmitted live to the other player, subjects can dramatically increase their success rates in the common interest games compared to the situation in which they do not see gaze. In the hide \& seek game, the seekers' success rate is the same as when they do not see gaze. Although aggregate success rates are consistent with gaze being completely uninformative, an analysis of individual gazing strategies suggests that there is heterogeneity in the chosen strategies. In the hide \& seek game, we identify two general types of strategic gaze:

\footnotetext{
Footnote 4 (continued)

minute before they burst out laughing at practically the same instant. 'My message was, 'Tonight I'd like to invite you to have supper on the Champs-Elysees,' he said. She doubled over with laughter. 'Mine was, 'You ... need to shave!'," (Liu 2008, p. 179).

5 People's gaze may involuntary reflect the decision-making process or be drawn to salient features. After all, professional poker players often wear sunglasses, eye movements of amateur blackjack players can reflect the numerical value of their hands (Holmes et al. 2016), and it is very difficult to prevent recognition of previously seen faces from being shown in the eyes (Schwedes and Wentura 2012).
} 
Most people try to mask their intended choice with an uninformative gaze pattern while some try to actively mislead their counterparts into holding a wrong belief. Some strategies to actively mislead the other player are more successful: In our data, hiders tend to be more successful when they do not choose the option they emphasize with their gaze.

Our results are a first step to explore how much communication, voluntary or involuntary, is possible with gaze. Gaining a better understanding of the strategic use of eye-movements is relevant for many economic interactions that take place in face-to-face meetings, but also more generally in all situations in which information search patterns can be observed. For instance, our results imply that software design (e.g. privacy settings) has to take into account that behavior changes if information search patterns are observed by others. Similarly, researchers should be aware that eye movements, and potentially also other biometrical measures, might be strategically altered depending on the context.

The remainder of the paper is organized as follows. Section 2 describes the experimental design and the hypotheses. The results are presented in Sect. 3, where we first focus on non-strategic gaze and then analyze whether and for which games gaze and success rates change when gaze is transmitted live instead of recorded. Finally, focusing on the hide \& seek game, we explore the success of different strategies and the heterogeneity of first mover behavior.

\section{Methods}

\subsection{Games}

All participants play four different two-player games: A coordination, a focal coordination, a focal discoordination, and a hide \& seek game. Each game is played for five consecutive rounds with random re-matching of participants and no feedback between rounds. The order of games is counterbalanced within each session. In all 20 rounds, both players see four large, gray squares arranged in a square on their computer screens and have to pick one of them. Subjects make their choices by pressing one of four keys marked with stickers ( R, U, C, and N, approximately mirroring the positioning of the boxes on the screen).

Participants' earnings are determined by whether or not the two players' actions match: In the coordination and the focal coordination game, both participants only earn points if they choose the same box. In the focal discoordination game, both participants only earn points if their actions do not match. In the hide \& seek game, the first mover earns points if the actions do not match whereas the second mover earns points if the actions do match.

The maximum number of points a player can earn by choosing a box is always displayed in the center of a box. In the coordination game and hide \& seek game, this number is always 10 in all four boxes (Fig. 1, left panel). In the focal coordination and focal discoordination game, only the top left box gives 10 points whereas the other three boxes give 11 points. Figure 1 (right panel) shows the decision screen for these games. 

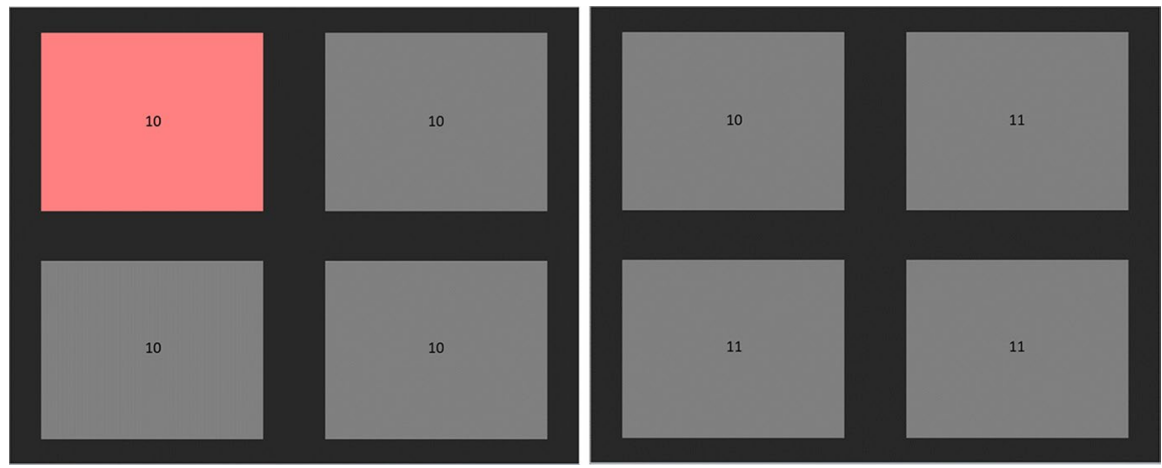

Fig. 1 Decision screen. The left panel shows a screenshot of a subject currently looking at the top left box, in either the coordination or the hide and seek game. The right panel shows the decision screen for the focal coordination and discoordination game without any box being looked at. For ease of readibility, we increased the font size of the numbers

\begin{tabular}{|c|c|c|c|}
\hline TL & TR & BL & BR \\
\hline 10,10 & 0,0 & 0,0 & 0,0 \\
\hline 0,0 & 10,10 & 0,0 & 0,0 \\
\hline 0,0 & 0,0 & 10,10 & 0,0 \\
\hline 0,0 & 0,0 & 0,0 & 10,10 \\
\hline
\end{tabular}

(a) Coordination Game

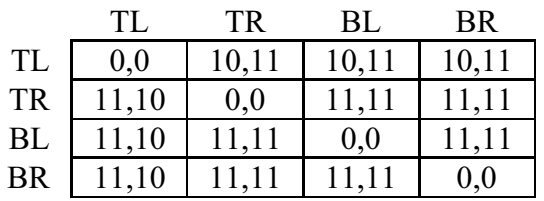

(c) Focal Discoordination Game

\begin{tabular}{l|c|c|c|c|}
\multicolumn{1}{c}{} & \multicolumn{1}{c}{ TL } & \multicolumn{1}{c}{ TR } & \multicolumn{1}{c}{ BL } & \multicolumn{1}{c}{ BR } \\
\cline { 2 - 5 } TL & 10,10 & 0,0 & 0,0 & 0,0 \\
\cline { 2 - 5 } TR & 0,0 & 11,11 & 0,0 & 0,0 \\
\cline { 2 - 5 } BL & 0,0 & 0,0 & 11,11 & 0,0 \\
\cline { 2 - 5 } BR & 0,0 & 0,0 & 0,0 & 11,11 \\
\cline { 2 - 5 } & & & &
\end{tabular}

(b) Focal Coordination Game

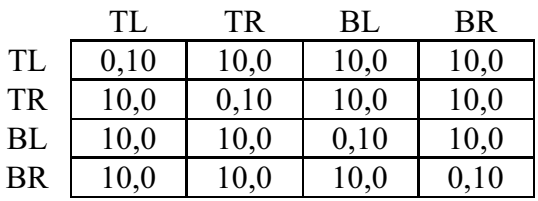

(d) Hide \& Seek Game

Fig. 2 The four games in normal form. Notes: The decision screens of the participants do not show the normal form, but a graphical representation of their possible choices (see Fig. 1). The strategies' labels TL, TR, BL, and BR in the normal form correspond to the top left, top right, bottom left, and bottom right box on the participants' computer screens, respectively

The rules of the games are made common knowledge to the participants (see Online Appendix for a translation of the instructions and all data files). Figure 2 summarizes the four games in normal form, which however seems less natural than the verbal description of the game and was therefore not part of the explanation of the games to the participants.

In all games, all possible individual choices are consistent with some Nash equilibrium. The coordination games have the very intuitive best response to choose the same strategy as the other player. There are four Nash equilibria in pure strategies in the coordination game, but strategies are isomorphic with no reason to choose one over the others except possibly the labeling of the strategies, which here corresponds to the boxes' locations. There is also a completely mixed equilibrium, in which every box is chosen with equal probability. 
Similar arguments apply to the focal coordination game, but here the strategies are not entirely isomorphic, since coordination on TL yields a slightly lower payoff. Thus, the completely mixed Nash equilibrium involves playing TL with slightly larger probability. Although the focal discoordination game is also symmetric, the only symmetric equilibrium involves completely mixed strategies which put slightly less probability on the less efficient focal option TL. Finally, the hide \& seek game is an asymmetric game which has a unique Nash equilibrium in mixed strategies with both players choosing all strategies with equal probability.

\subsection{Treatments}

We use four between-sessions treatments to explore the role of gaze: NoGaze, RecordedGaze, LiveGaze-FreeChoice, and LiveGaze-ForcedChoice. In NoGaze, the gaze of the first mover is recorded but not shown to the second mover. With this benchmark treatment, we can investigate the informativeness of non-strategic gaze. In RecordedGaze, this earlier recorded non-strategic gaze data is displayed to subjects who then also play against the corresponding old decisions. This allows us to infer how well subjects understand non-strategic gaze.

In the two remaining treatments, the first mover's gaze is transmitted in real time to the second mover, which allows studying the communicative function of gaze. In LiveGaze-FreeChoice, the first mover is able to freely choose any of the four boxes. To disentangle the effect of choices and gaze on achieved payoffs, we introduce the LiveGaze-ForcedChoice treatment. Here, first movers only earn points if the chosen box coincides with the one that has been randomly selected by the computer beforehand. This box is marked by an arrow that is only visible to the first player (and not to the other player) for two seconds before each round. In the LiveGaze-ForcedChoice treatment, subjects do not have to think about strategic choices and can focus on the strategic use of gaze, and saliency no longer serves as a coordination device.

\subsection{Procedures}

We visualize gaze by changing the color of the square that is currently looked at by the first mover from gray to red (see Fig. 1, left panel). This is the case on both the screen of the first mover and on the screen of the second mover, i.e. the first mover is fully aware of how his gaze data is displayed to the second mover. Although no gaze data is transmitted between players in NoGaze, we nevertheless keep the treatments as symmetric as possible: For the first movers, the boxes still change color according to where they look as in the LiveGaze treatments. Likewise, a second mover also has to wait for the first mover to enter his decision before she can enter hers-she merely does not see any gaze data.

Participants enter their decisions sequentially without observing the other player's choice. The eye-tracked player chooses first. Once the first mover enters his decision, a green frame appears around the second mover's screen indicating to her that she can now enter her decision. Before each round, subjects see a brief reminder of the payoff consequences of matching and non-matching choices for both players 
for eight seconds, followed by a two-second display announcing the start of the next round, and finally a fixation cross in the center of the screen for one second.

To account for our method of gaze visualization in the analysis, we deviate from common fixation definitions which typically require that the position of gaze remains constant for some minimum amount of time (see Salvucci and Goldberg (2000)). Instead, we consider everything a fixation that is actually displayed to the participants. A fixation thus starts the moment the respective box turns red and lasts until the box turns gray again.

We used Tobii EyeX ${ }^{6}$ eye trackers with a sampling rate of $60 \mathrm{~Hz}$. Subjects sat approximately $58 \mathrm{~cm}$ in front of their screens, all of which were of the same model and had a resolution of $1280 \times 1024$ pixels. To improve data quality, we used chin rests for all eye-tracked subjects. None of the subjects had issues with the eye-trackers.

We ran three sessions in each treatment with eye-tracking (NoGaze, LiveGazeFreeChoice, and LiveGaze-ForcedChoice), each with 24 subjects of which the twelve first movers were eye-tracked. Data acquisition for the RecordedGaze treatment was finished within one session with 30 second movers who played against the recorded gaze and choices of first movers from the NoGaze treatment. Recall that subjects were randomly rematched after each round such that each second mover faced a unique sequence of first movers. Thus, each second mover in RecordedGaze faced one of the exact sequences of first movers that was previously faced by a second mover in the NoGaze treatment. The sequences were chosen at random without replacement.

The experiment was programmed in z-Tree (Fischbacher 2007) and our subjects were recruited from the general student population of the University of Konstanz using the software ORSEE (Greiner 2015). Each session lasted around $1 \mathrm{~h}$, and subjects were paid $€ 0.1$ for each point they earned in the experiment such that they received an average of around $€ 17$. Our participants had an average age of about 21 years and about $40 \%$ were male.

\footnotetext{
${ }^{6}$ Gibaldi et al. (2017) extensively test the Tobii EyeX and find that this eye tracker is suitable for some research but not for tasks which need a higher sampling rate. They find the accuracy is between 0.5 and $1^{\circ}$ while the precision is at $0.25^{\circ}$. Tobii states that no precision above $1.5^{\circ}$ will pass a calibration routine. Given our distance from the screen, the font size and the size of the rectangles $(500 \times 400$ pixels $)$, we should detect all gazes on the number. Further, each box is 70 pixels in the horizontal and 56 pixels in vertical dimension apart from the end and middle of the screen. Therefore, the distance between boxes is 140 pixels $\left(4.7^{\circ}\right)$ in horizontal and $112\left(4.1^{\circ}\right)$ in vertical dimension, which should be sufficient to rule out that the wrong box changes the color. Note that it is not necessarily important for us that subjects look at the number (they were mostly the same), but we wanted every subject to have a reasonable calibration quality and that all subjects participate. For these reasons the Tobii EyeX presents an appropriate apparatus. All subjects see which field is highlighted and could adjust if necessary. Eye-blinks or closing the eyes, lead to no change in the status quo of the displayed gaze, i.e. if a person looks at the TL box and closes her eyes, the TL box is still red.
} 


\subsection{Hypotheses}

With this design, we aim at answering the following questions: First, how informative is gaze about choices and to what extent are subjects able to exploit this information? Second, how do the answers to these questions depend on the strategic situation? Several studies have shown that decisions often follow gaze, in the sense that the chosen option is both the one that was looked at last and for a longer time (see, e.g. the gaze cascade effect as described by Shimojo et al. (2003), or the attentional drift diffusion model by Krajbich et al. (2010)). Gaze should therefore contain some information about strategy choice in a game. Hypothesis 1 ("Interpreting gaze") states that it is possible to infer strategy choices from the observed gaze of the choosing subject.

Following the literature mentioned above, we will use mainly two pieces of information in order to interpret gaze: Which box first movers looked at for the longest time or last. If Hypothesis 1 is true, then these criteria (Most Looked and Last Looked) should already reliably predict choice in the RecordedGaze treatment. How well people are able to interpret gaze is measured by the second mover's success rate in RecordedGaze compared to NoGaze.

Our second hypothesis is concerned with how the eye-tracked players react to gaze being observed by the second mover. It is based on the assumption that gaze can be used as a communication device to announce a strategy choice, e.g. by focusing on one option for a long time. Hence, Hypothesis 2 ("Strategic gazing") states that subjects are aware that their gaze conveys information and are able to strategically adapt it.

If people are able to infer meaning from another person's gaze, then our setup essentially adds a prior communication stage to the game. Communication via gaze is cheap talk in the sense that it does not directly affect payoff. Nevertheless, if being predictable is in a player's self-interest, then she should try to communicate her intentions as clearly as possible. In the common interest games, communicating one's intended choice is "self-signaling" and "self-committing": A player wants the other to think that she will choose a strategy if and only if she indeed intends to choose it, and if a player has persuaded the other player that she will play a certain strategy, she indeed wants to choose that strategy (see, e.g. Farrell and Rabin (1996)).

If instead the players have opposed interests, as in the hide \& seek game, then only the "babbling" equilibrium, in which all messages are ignored, exists. In this game, all communication must be completely uninformative in equilibrium. Hence, if Hypothesis 2 is true, second movers' success rates should be higher in the common interest games for the LiveTreatments than for the NoGaze and RecordedGaze treatment. In contrast, the second mover's success rate in the hide \& seek game should be highest in RecordedGaze, while it should be close to chance in LiveTreatments and in NoGaze.

The two different LiveGaze treatments allow to distinguish whether observed success rates are due to players being attracted to salient choices, or due to information content of gaze alone. Hence, if in any game the second movers' baseline (NoGaze) success rates are a lot higher (or lower) than chance, then we may expect success 
rates to be higher (lower) in LiveGaze-FreeChoice than in LiveGaze-ForcedChoice. This may matter in particular if it should turn out that baseline success rates are already so large that they are difficult to improve upon.

In the games with a focal option, the LiveGaze-FreeChoice treatment is expected to have an impact on choices when there is an inferior focal option. The top left box may attract gaze because it stands out with a lower number of points but also because the western reading style is used to starting in the top left corner of a text (Abed 1991). The top left box can be chosen in equilibrium when communication is impossible, but not anymore once intended choice can be signaled with gaze. ${ }^{7}$

The common interest games differ in their complexity and thus may shed some light on the limits of signaling one's intentions through gaze. A focal option that players want to avoid may distract gaze, although it is believed that focal points are no longer important once communication is possible (Farrell and Rabin 1996). In the discoordination game, it becomes relevant that gaze is not a rich natural language (in the sense of Farrell (1993)), since not all messages can be unambiguously conveyed with gaze. In contrast to the coordination games, it matters now whether focusing on e.g. the top right box is understood as "I will choose the top right box" or "You should choose the top right box". 8

These arguments lead to the following order of the signal quality between games and treatments: The chosen option should be clearly communicated with gaze (better than in RecordedGaze) in the common interest games, with the pure coordination game being the easiest, then the focal coordination, and finally the focal discoordination game. Gaze in the hide \& seek game should on average reveal close to nothing about intended choices.

Our predictions are targeted at aggregate statistics. However, since we observe initial behavior without feedback between rounds, gazing strategies may differ from equilibrium and across subjects. We exploratively investigate two different gazing strategies: Hiding all information from one's gaze (signal-jamming) and trying to actively mislead the opponent into holding a wrong belief. Individual gazing strategies are addressed in more detail at the end of Sect. 2.

\section{Results}

\subsection{Aggregate choices}

To get a first impression of the effect of transmitting gaze, we start the analysis with a brief description of the choice distributions across treatments (Table 1). Despite the symmetric layout of the four boxes in a square, the choice data from the coordination game in NoGaze reveals a tendency to favor the top boxes. A $\chi^{2}$-test rejects

\footnotetext{
7 In games with perfectly aligned interests, a player can always use a common language to send a credible message (like pointing with gaze here) to coordinate on a Pareto efficient outcome.

${ }^{8}$ See also Ellingsen and Östling (2010) for this distinction between communication protocols that induce cheap talk about promises of own actions or requests about other's actions.
} 
Table 1 Average box choices in treatments with free choice

\begin{tabular}{lclll}
\hline & Coordination & Focal Coordination & Focal Discoordination & Hide \& Seek \\
\hline NoGaze & & & & \\
Top left & $24 \%(31 \%)$ & $35 \%(59 \%)$ & $18 \%(27 \%)$ & $22 \%(24 \%)$ \\
Top right & $47 \%(32 \%)$ & $38 \%(18 \%)$ & $23 \%(17 \%)$ & $17 \%(29 \%)$ \\
Bottom left & $9 \%(21 \%)$ & $9 \%(11 \%)$ & $32 \%(28 \%)$ & $32 \%(21 \%)$ \\
Bottom right & $19 \%(17 \%)$ & $17 \%(12 \%)$ & $27 \%(28 \%)$ & $29 \%(26 \%)$ \\
RecordedGaze & & & & \\
Top left & $27 \%(27 \%)$ & $35 \%(25 \%)$ & $18 \%(17 \%)$ & $23 \%(27 \%)$ \\
Top right & $44 \%(41 \%)$ & $38 \%(42 \%)$ & $23 \%(27 \%)$ & $18 \%(19 \%)$ \\
Bottom left & $11 \%(13 \%)$ & $9 \%(11 \%)$ & $34 \%(27 \%)$ & $31 \%(30 \%)$ \\
Bottom right & $19 \%(20 \%)$ & $18 \%(21 \%)$ & $25 \%(29 \%)$ & $29 \%(25 \%)$ \\
LiveGaze-FreeChoice & & & & \\
Top left & $34 \%(36 \%)$ & $1 \%(1 \%)$ & $12 \%(10 \%)$ & $26 \%(29 \%)$ \\
Top right & $37 \%(36 \%)$ & $46 \%(46 \%)$ & $34 \%(23 \%)$ & $16 \%(27 \%)$ \\
Bottom left & $13 \%(12 \%)$ & $17 \%(18 \%)$ & $29 \%(35 \%)$ & $34 \%(24 \%)$ \\
Bottom right & $17 \%(16 \%)$ & $36 \%(36 \%)$ & $24 \%(32 \%)$ & $23 \%(20 \%)$ \\
\hline
\end{tabular}

The table shows the choice distributions of the eye-tracked participants and those of the second movers in parentheses. There were 36 first and second movers in the NoGaze and the LiveGaze-FreeChoice treatments, 30 second movers in the RecordedGaze treatment. Data of first movers in the RecordedGaze treatment stems from the 30 randomly selected first movers from the NoGaze treatment. Each participant played each game five times. Totals unequal $100 \%$ are due to rounding

the null-hypothesis that first movers are choosing randomly $\left(\chi^{2}=26.5\right.$ and $p<0.001$ for first movers and $\chi^{2}=6.42$ and $p<0.1$ for second movers). Choices in the focal coordination game are similarly more concentrated on the top boxes, with the focal top left box being chosen more often. ${ }^{9}$ Hence, the lower payoff in the top left box indeed has the effect of making this option more salient.

In the discoordination and hide \& seek game, in which the first mover receives a payoff if the second mover does not choose the same box, this pattern is reversed in that the bottom boxes are chosen more frequently. However, these distributions are not significantly different from a uniform distribution. The finding that the top boxes are chosen less frequently by the first movers is in line with the finding by Rubinstein et al. (1997) that hiders choose the apparently salient option less often than seekers.

A comparison of choices across treatments already suggests that gaze transfer helps to coordinate: When they can see recorded gaze, the second movers' choice

\footnotetext{
${ }^{9}$ Such a focal coordination game was described by Schelling (1960) and later used in an experiment by Bardsley et al. (2010) to study how focal points can foster coordination. Our data fits in with their conclusion that both "level-k reasoning" and "team reasoning" seem to occur in this game. Nine subjects switch to a predominant choice of the top left box in the focal coordination game, which is only consistent with team reasoning, but there are also six subjects who never choose the top left box in the focal coordination game.
} 


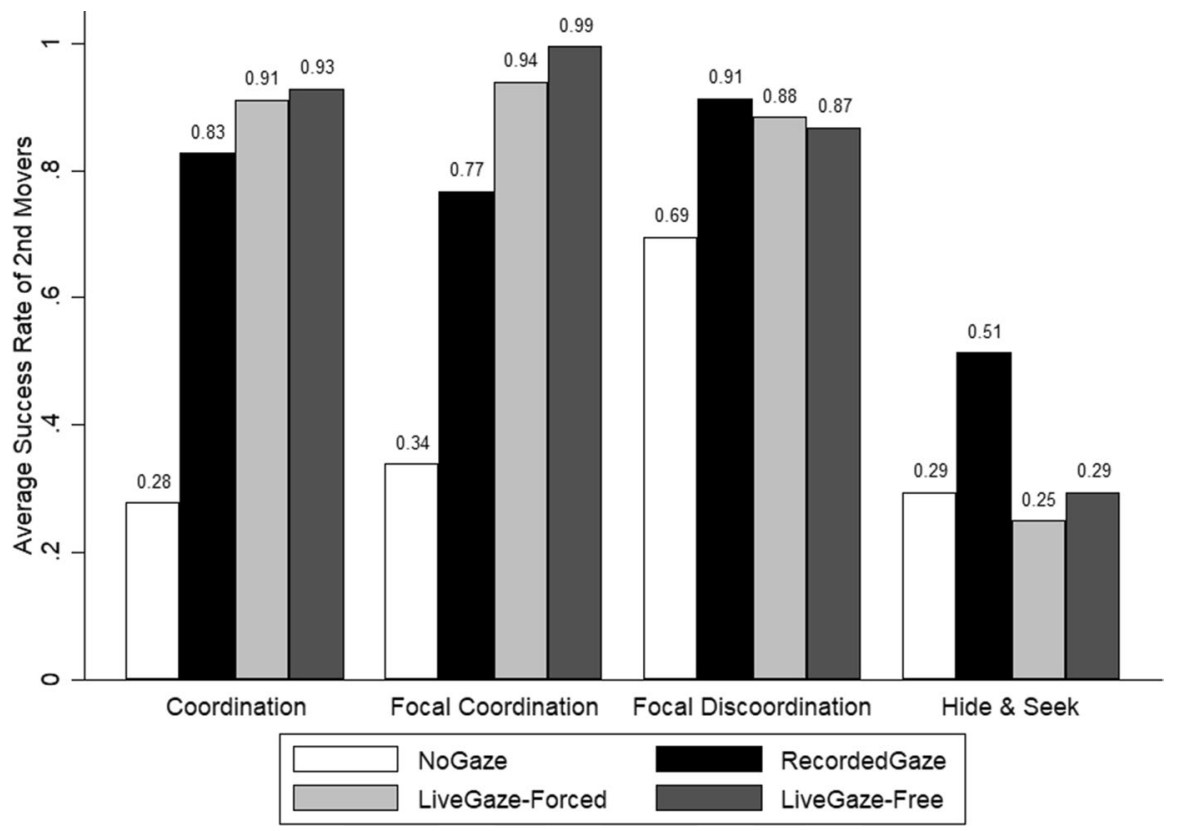

Fig. 3 Average success rates of second movers across treatments and games. Notes: A trial is counted as a success for the second mover when she receives a positive payoff, i.e. when the two players choose the same box in the coordination, the focal coordination, and the hide \& seek game and when they choose different boxes in the focal discoordination game

distribution is similar to that of the first movers in both coordination games. This is even more pronounced for live gaze transmission. Moreover, in LiveGaze-FreeChoice, the inefficient outcome in the focal coordination game is avoided almost completely by the players. In the focal discoordination game, the inefficient option is still chosen in LiveGaze-FreeChoice, but less often.

\subsection{Non-strategic gaze}

We now investigate how well subjects understand non-strategic gaze (RecordedGaze). Figure 3 depicts the average success rates of the second movers across all four treatments and games. Participants in RecordedGaze indeed considerably gain from seeing the first movers' gaze previously recorded in NoGaze. Second movers in RecordedGaze achieve significantly higher success rates in all four games than in NoGaze (Wilcoxon rank-sum tests comparing individual average success rates in each game by treatment, all Bonferroni corrected $p$ values $<0.03$ ). In the three common interest games, second movers do remarkably well in interpreting the recorded gaze patterns. Although the success rate in the hide \& seek game is also significantly higher when non-strategic gaze can be observed, the gained advantage is less pronounced. 
Table 2 Shares of correct predictions by the two heuristics in the recorded gaze treatment

\begin{tabular}{lllll}
\hline & Coordination & Focal Coordination & Focal Discoordination & Hide \& Seek \\
\hline Most Looked & 0.82 & 0.89 & 0.82 & 0.61 \\
Last Looked & 0.94 & 0.92 & 0.88 & 0.73 \\
\hline
\end{tabular}

Note that for all games except the focal discoordination game, the share of correct predictions corresponds to the second movers' success rate which they would have achieved if they had all followed the predictions of the respective heuristic. For the discoordination game, even when making the wrong prediction about choice, there is still a $2: 1$ chance to discoordinate

The second mover in the hide \& seek game has to guess the choice of the first mover, which is exactly the same task, with the same decision screen, as in the coordination game. Hence, the first mover's gaze must be more difficult to interpret in this game. As mentioned in the Hypotheses Section, we use two simple heuristics in order to interpret gaze: The Most Looked heuristic predicts that the first mover chooses the box that he looks at for the longest time and the Last Looked heuristic states that he chooses the box that he looks at last. We compare the predictive success of these two rules in order to show how the gaze data reveals less about choices in the hide \& seek game.

Table 2 shows the average success rates the second movers in the RecordedGaze treatment would achieve if they all followed the respective heuristics. The heuristics also perform worse in the hide \& seek game than in the other games (pairwise Wilcoxon rank-sum Bonferroni corrected $p$ values $<0.002$ except for the predictive success of the Last Looked heuristic in the hide \& seek vs. the focal discoordination game with corrected $p=0.072$ ). Hence, gaze indeed seems to be less informative about the actual choice in the hide \& seek game, which can explain why the second movers in the RecordedGaze treatment profit least from seeing the gaze in the hide $\&$ seek game. In the common interest games, the two heuristics correctly predict the chosen box most of the time. In all games, the heuristics, especially the Last Looked heuristic, perform a bit better than the participants did.

The top part of Table 3 shows the shares of participants choosing in line with each heuristic. There is also a difference between the hide \& seek and the other games here: second movers do not choose as often according to the heuristics in the hide \& seek game.

Summarizing the results of the RecordedGaze treatment, we find strong support for our first hypothesis: People are able to correctly interpret gaze that is purely instrumental. Contrary to our expectations, the increase in the success rate depends on the strategic nature of the game: Choice in the competitive hide \& seek game is harder to predict from non-strategic gaze than in the common interest games.

\subsection{Strategic gaze: success rates}

We now turn to the analysis of strategic gaze. When comparing RecordedGaze and the two LiveGaze treatments in Fig. 3, we can see that subjects increase their 
Table 3 Share of second mover choices in line with heuristics by treatment

\begin{tabular}{lllll}
\hline & Coordination & Focal Coordination & Focal Discoordination & Hide \& Seek \\
\hline $\begin{array}{l}\text { RecordedGaze } \\
\text { Most Looked }\end{array}$ & 0.82 & 0.77 & & 0.68 \\
Last Looked & 0.86 & 0.81 & 0.94 & 0.68 \\
LiveGaze-ForcedChoice & & & & 0.46 \\
Most Looked & 0.94 & 0.96 & 0.93 & 0.44 \\
Last Looked & 0.94 & 0.94 & 0.92 & \\
LiveGaze-FreeChoice & & & & 0.31 \\
Most Looked & 0.96 & 0.99 & 0.93 & 0.30 \\
Last Looked & 0.94 & 0.99 & 0.92 & \\
\hline A & & & & \\
\hline
\end{tabular}

A second mover's choice is counted as in line with the heuristic, if it is a best response to the heuristic's predicted choice of the first mover. Note that, since the heuristics always make point predictions for the first mover, there are always three best responses in the focal discoordination game whereas there is only one in all other games

success rate even further when the first movers' gaze is live transmitted to their current partner in the two coordination games. In the coordination game, the success rates significantly increase from $83 \%$ in RecordedGaze to $91 \%$ and $93 \%$ in LiveGaze-ForcedChoice and LiveGaze-FreeChoice (pairwise Wilcoxon rank-sum tests comparing individual average success rates by treatment, both corrected $p$ values $<0.04)$. In the focal coordination game, the effect is even more extreme: The success rate of $77 \%$ in RecordedGaze increases to $94 \%$ in LiveGaze-ForcedChoice and even to $99 \%$ in LiveGaze-FreeChoice (both corrected $p$ values <0.04).

However, in the focal discoordination game, the success rates are slightly lower in LiveGaze than in RecordedGaze, but these differences are non-significant (both uncorrected $p$ values $>0.4$ ). Despite the slight decrease in the focal discoordination game, live gaze transmission nevertheless leads to significantly higher success rates compared to NoGaze (both corrected $p$ values $<0.001$ ). Thus, by using their gaze as a signaling device, first movers are clearly able to make themselves more predictable than without gaze transmission in all three common interest games.

This pattern changes in the hide \& seek game: The success rates in the two LiveGaze treatments (25\% and 29\%) are both significantly lower than in RecordedGaze $(51 \%$, both corrected $p$ values $<0.01)$. Hence, first movers are on average able to hide their intentions when it is in their interest to become less predictable. However, they are not able to increase their success rate compared to NoGaze by somehow misleading seekers with their gaze. In fact, the success rate in LiveGaze-FreeChoice is the same as in NoGaze and the success rate in LiveGazeForcedChoice is the one that would result in a hypothetical NoGaze treatment with random choices. These are the predicted rates if gaze was completely uninformative. The difference between these two success rates is non-significant (both uncorrected $p$ values $>0.3$ ). 
Table 4 Average fixation numbers and fixation durations across games and treatments

Coordination Focal Coordination Focal Discoordination Hide \& Seek

$\begin{array}{lcccc}\text { Average number of fixations } & & & & \\ \text { NoGaze } & 5.3 & 5.3 & 4.6 & 5.3 \\ \text { LiveGaze-ForcedChoice } & 2.8 & 2.6 & 5.3 & 9.3 \\ \text { LiveGaze-FreeChoice } & 4.7 & 3.4 & 4.8 & 13.8 \\ \begin{array}{l}\text { Average fixation duration in } \\ \quad\end{array} & & & \\ \quad \text { seconds } & 1.05 & 0.83 & 1.00 & 0.66 \\ \text { NoGaze } & 2.57 & 2.67 & 1.84 & 0.67 \\ \text { LiveGaze-ForcedChoice } & 1.85 & 2.43 & 1.95 & 0.73 \\ \text { LiveGaze-FreeChoice } & & & \end{array}$

The top half of the table shows the average number of fixations between boxes per game for each treatment. The bottom half shows the according average fixation durations in seconds. We consider everything a fixation that is displayed to the participants as a red box, i.e. a fixation starts when the respective box turns red and ends when it turns gray again

\subsection{Strategic gaze: characteristics}

To get an impression of how first movers make use of the opportunity to gaze strategically, we compare the average number of fixations and the average fixation durations across games (see Table 4). The gaze characteristics do not differ much in the NoGaze treatment or between the three common interest games in the LiveGaze treatments. In contrast, the average number of fixations is significantly higher in the hide \& seek game compared to the three common interest games in both LiveGaze treatments (pairwise Wilcoxon signed ranks tests, all corrected $p$ values $<0.02$ ). Similarly, the average fixation duration is significantly shorter in the hide $\&$ seek game than in the other three games for both LiveGaze treatments (all corrected $p$ values $<0.01$ ). Thus, subjects move their gaze more quickly from one box to another and do so more often in the hide \& seek game than in the common interest games, which makes the gaze more difficult to interpret.

These effects also become apparent in the predictive success of the two heuristics we introduced earlier (Most Looked and Last Looked, see Table 5). In both LiveGaze treatments, the success rates of both heuristics in the hide \& seek game drop to values of $31 \%$ and below. This suggests that at least some first movers actively try to keep their gaze from the chosen option. In contrast, in the coordination as well as the focal coordination game, the heuristics' hit rates lie between 96 and 100\%, indicating that the first movers succeed in sending a clear signal. Finally, first movers seem to have more difficulties in sending clear signals in the focal discoordination game as the heuristics' success rates only lie between 74 and $82 \%{ }^{10}$

\footnotetext{
10 The success rates of all heuristics are significantly smaller in the hide \& seek game than in all other games (Wilcoxon rank-sum tests, all corrected $p$ values $<0.002$ ). Further, success rates in the focal discoordination game are significantly smaller than in the (focal) coordination games (all corrected $p$ values $<0.002$ ), and they do not significantly differ between the focal and the non-focal coordination game (all corrected $p$ values $>0.1$ ).
} 
Table 5 Shares of correct predictions by the two heuristics in live gaze treatments

\begin{tabular}{lllll}
\hline & Coordination & Focal Coordination & Focal Discoordination & Hide \& Seek \\
\hline LiveGaze-ForcedChoice & & & & \\
Most Looked & 0.97 & 0.96 & 0.74 & 0.22 \\
Last Looked & 0.97 & 0.99 & 0.79 & 0.31 \\
LiveGaze-FreeChoice & & & & 0.26 \\
Most Looked & 0.96 & 1.00 & 0.79 & 0.30 \\
Last Looked & 0.97 & 1.00 & 0.82 & \\
\hline
\end{tabular}

The table shows each heuristic's share of correct predictions in each game for the LiveGaze-ForcedChoice (top) and the LiveGaze-FreeChoice treatment (bottom)

The heuristics' hit rates in the focal coordination game in LiveGaze-FreeChoice are exceptionally high (100\% for the both Last Looked and Most Looked heuristic). This indicates that the presence of the salient but inefficient outcome in the focal coordination game makes subjects exhibit the most telling gaze. They clearly avoid looking at the focal option: Compared to the coordination game, the share of first fixations on the top left box drops from more than $50 \%$ to less than $15 \%$ in the focal coordination game. Similarly, the share of trials in which the top left box is not even looked at once increases from 36 to $71 \%$. Hence, it is not the case that the presence of a focal option makes it more difficult to communicate choice of another box with gaze.

In the focal discoordination game, the heuristics' hit rates are significantly lower than in the other common interest games. This indicates that the ability to send clear signals also depends on the nature of the strategic situation at hand. A discoordination game adds complexity to the situation as the signal becomes ambiguous: It could both be an announcement of the first mover's own strategy but also a choice recommendation for the second mover. This aspect could make it harder for the first movers to commit to a particular gazing strategy such that their gaze becomes more difficult to interpret. Further, the share of second movers deciding in line with a belief based on the respective heuristics never drops below $90 \%$ also in the focal discoordination game (see Table 3). This indicates that the failure to increase the success rate in the discoordination game with strategic compared to non-strategic gaze is due to first movers not choosing the box that they emphasized with their eye gaze.

In order to explore the effects of the strategic environment on the signal quality more closely, we also analyze the distribution of viewing time across the four boxes. Figure 4 shows the cumulative distribution of the share of viewing time which subjects allocate to all but the most inspected box (separately for all four games and the two LiveGaze treatments). The intercept with the $y$-axis indicates the share of games in which the respective first mover only looked at a single box. This share is largest in the focal coordination game in both LiveGaze treatments $(86 \%$ and $67 \%$, 


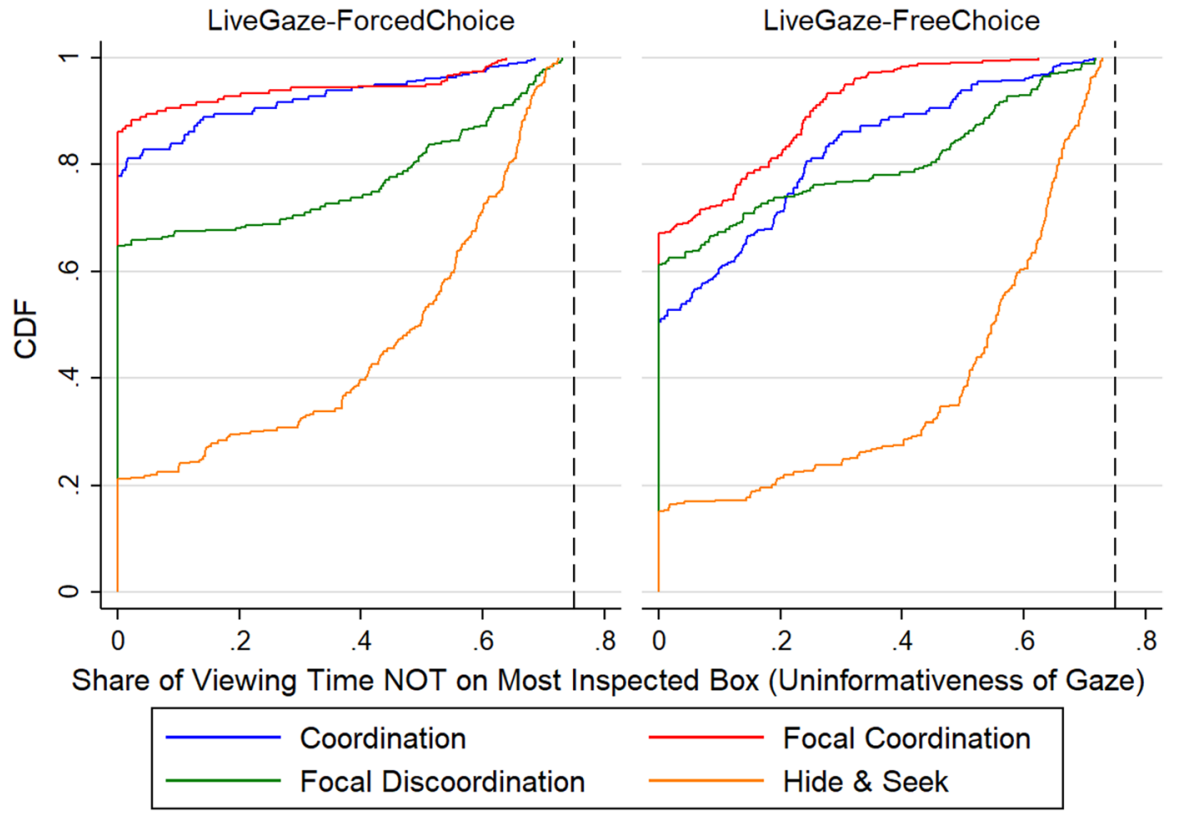

Fig. 4 Cumulative distribution of share of viewing time on all boxes other than the most inspected box across games and livegaze-treatments. Notes: The graphs show the distribution of the share of viewing time that subjects allocate to all boxes except the one they inspect the most. The intercept with the $y$-axis thus corresponds to the percentage of people that only look at one single box in the respective game and treatment. The dashed lines mark the natural maximum of $75 \%$ viewing time on the non-most inspected boxes, which is reached if all four boxes are inspected for exactly $25 \%$ of the time

respectively). In LiveGaze-FreeChoice, the cumulative distribution of gaze time in the focal coordination game is always to the left of that in the coordination game, i.e. gaze in the focal coordination game is particularly clear also with respect to the allocation of viewing time (two-sided Kolmogorov-Smirnov test, corrected $p$ value $<0.001$ ).

This is different for the focal discoordination game. In both treatments, the share of first movers looking at only one box is lower in the focal discoordination game than in the focal coordination game. The cumulative distributions of gaze time in the focal discoordination game are also always to the right of their counterparts from the focal coordination game and these differences are significant (Kolmogorov-Smirnov tests, both corrected $p$ values $<0.001$ ). Signal strength in terms of viewing time thus clearly suffers in the focal discoordination game. In the hide \& seek game, the share of viewing time of all but the most looked-at choice start out relatively low and flat and becomes steeper toward the dashed lines. This indicates that at least some first movers looked at each of the boxes for approximately the same amount of time, which blurs their intended choice. 


\subsection{Strategic gaze in hide \& seek: signal-jamming and misleading}

We now look at the success rates of different gaze-choice combinations across all trials in the hide \& seek game. Among the gaze strategies in which all four boxes are looked at, the mean number of fixations is in fact very high in both LiveGaze treatments (13.8 in ForcedChoice and 18.4 in FreeChoice), indicating that these first movers are indeed trying to make themselves unpredictable. The modal gaze in both LiveGaze treatments is characterized by this method $56.7 \%$ in ForcedChoice and $66.1 \%$ in FreeChoice, see Table 6). ${ }^{11}$

A second straightforward method to hide one's intended choice in our setting would be to simply not look at any boxes at all, e.g. by closing your eyes and blindly pressing a button. We did not mention this possibility in the instructions but we neither explicitly forbade it. Nevertheless, only $6.1 \%$ of trials feature this method in LiveGaze-ForcedChoice and only 8.9\% in LiveGaze-FreeChoice.

Apart from trying to hide their intentions, first movers could also try to actively mislead second movers into holding a wrong belief. To achieve this, some participants try to emphasize one or more boxes with their gaze and then choose either the emphasized or a different box. In the post-experimental questionnaire, participants using such techniques for instance describe them like this: "[I] never looked at the chosen box"; "[I looked] at the chosen box because I assumed that the respective other partner would not expect this"; "I concentrated on a different box and switched to another box shortly before my choice but also did not choose that one"; "I always looked at a different box first and then briefly at the one I chose and then at the first one again". In LiveGaze-ForcedChoice, $20 \%$ of choices are made by only looking at a single box and $17.2 \%$ looking at two or three boxes. Similarly, in LiveGazeFreeChoice, $13.9 \%$ of choices feature gaze at only one box and $11.1 \%$ feature gaze at two or three boxes. Table 6 summarizes which strategies are applied in the two LiveGaze treatments and shows their respective success rates in parentheses.

Recall that, on average, first movers in both LiveGaze treatments are able to reach success rates statistically indistinguishable from those in NoGaze (70.5\%). However, in LiveGaze-ForcedChoice, much higher success rates are reached if neither the box they inspect most nor last is chosen. Looking at only one box, but then choosing a different one, even reached an average success rate of $90 \%$. As can be seen in Table 7, this effect is also significant. It shows the marginal effects of several probit models of first movers' success in the hide \& seek game regressed on the different strategies. The baseline choice strategy that is left out in the models is choosing the most emphasized box (i.e. the box that is looked at last when this was also the box that is looked at most).

The top half of the table shows the results for LiveGaze-ForcedChoice. In the first column, we see that the probability of a first mover success increases by more than $30 \%$ if he chooses neither the last nor the most inspected box. Looking at the same model estimated for different sub-samples, we see that this effect is especially

\footnotetext{
${ }^{11}$ There are also a few first movers who in every round do not look at all four boxes: 7 in ForcedChoice and 4 in FreeChoice.
} 


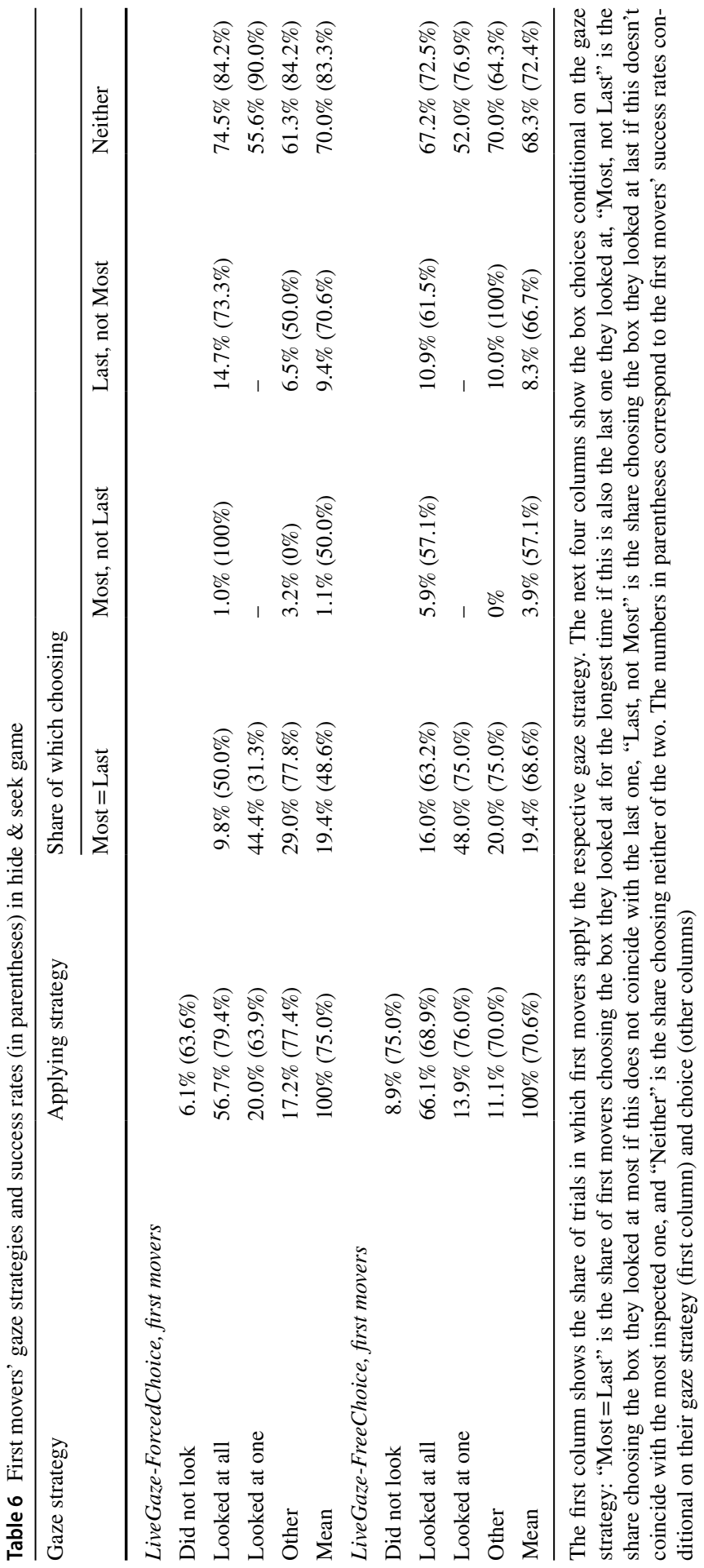


Table 7 First mover success regressed on gaze strategy (probit models, marginal effects)

Probit dependent variable: first mover success (binary)

\begin{tabular}{|c|c|c|c|c|}
\hline (Sub-)sample & Full & Looked at all & Looked at one & Other \\
\hline \multicolumn{5}{|c|}{ LiveGaze-ForcedChoice } \\
\hline Most, not Last & $\begin{array}{l}.010 \\
(.087)\end{array}$ & (omitted) & - & (omitted) \\
\hline Last, not Most & $\begin{array}{l}.140^{\dagger} \\
(.083)\end{array}$ & $\begin{array}{l}.117^{\dagger} \\
(.069)\end{array}$ & - & $\begin{array}{l}-.174 \\
(.435)\end{array}$ \\
\hline Neither & $\begin{array}{l}.337 * * \\
(.121)\end{array}$ & $\begin{array}{l}.283^{*} \\
(.128)\end{array}$ & $\begin{array}{l}.588 * * * \\
(.154)\end{array}$ & $\begin{array}{l}.145 \\
(.201)\end{array}$ \\
\hline $\mathrm{N}$ & 180 & 102 & 36 & 31 \\
\hline \multicolumn{5}{|c|}{ LiveGaze-FreeChoice } \\
\hline Most, not Last & $\begin{array}{l}-.104 \\
(.176)\end{array}$ & $\begin{array}{l}-.055 \\
(.197)\end{array}$ & - & (omitted) \\
\hline Last, not Most & $\begin{array}{l}-.018 \\
(.153)\end{array}$ & $\begin{array}{l}-.015 \\
(.173)\end{array}$ & - & (omitted) \\
\hline Neither & $\begin{array}{l}.038 \\
(.083)\end{array}$ & $\begin{array}{l}.091 \\
(.124)\end{array}$ & $\begin{array}{l}.019 \\
(.168)\end{array}$ & $\begin{array}{l}-.111 \\
(.314)\end{array}$ \\
\hline $\mathrm{N}$ & 180 & 119 & 25 & 18 \\
\hline
\end{tabular}

Baseline category is "Most = Last", i.e. choosing the box that was looked at most when it coincides with the box that was looked at last. Explanatory variables are dummies corresponding to the other choice strategies. The same model is estimated on different sub-samples. The whole sample was used in the models in the first column. In the second column, only those trials were included in which all four boxes were inspected at least once. In the third column, only those in which exactly one box was looked at and in the last column all trials in which two or three boxes were inspected. Robust standard errors clustered at the subject level. Standard errors for marginal effects in parentheses obtained via the Delta-Method. All omitted cells are due to too few observations in cell

$* * *$ Significant at the $0.1 \%$ level. **Significant at the $1 \%$ level. *Significant at the $5 \%$ level. Significant at the $10 \%$ level

pronounced for those subjects that only look at a single box but then choose a different one. These subjects even significantly increase their success probability by $58.8 \%$ compared to those that choose the only box they look at (column "Looked at One"). The effect of choosing neither the most nor the last inspected box is also significantly positive for those subjects who look at all four boxes at least once (+28.3\%, column "Looked at All"). For the same group of subjects, also choosing the box they look at last significantly increases their success probability by $11.7 \%$ when this box did not coincide with their most inspected box. ${ }^{12}$

In LiveGaze-FreeChoice, however, none of the strategies significantly affect the first movers' success probabilities, neither in the whole sample nor in any of the

\footnotetext{
12 More than $50 \%$ of second movers chose the box that the first mover either looked at most or last (or both) in the hide \& seek game in LiveGaze-ForcedChoice even when the first movers tried to blur their intention and looked at all four boxes (see Table 6).
} 
sub-samples. At least the tendencies are the same: Both when only one or when all boxes are looked at, success rates are highest when neither the most nor the last inspected box is chosen. It thus seems that although some people try to mislead their counterpart, only few succeed and only in specific circumstances. The focus on gazing in LiveGaze-ForcedChoice without having to worry about the actual choice (which is practically made by the computer) seems to help at least some subjects in choosing a good gaze strategy.

\subsection{Heterogeneity}

\subsubsection{Classification of types}

While our initial hypotheses were focused on the aggregate success rates, the previous section has shown that there is considerable heterogeneity in the strategies that subjects employ in the hide \& seek game. In this section, we give some structure to this analysis of individual behavior, asking how subjects with different levels of strategic sophistication behave in the different games. We rely on the "level-k" model of strategic thinking (Nagel 1995; Stahl and Wilson 1995; Ho et al. 1998; CostaGomes et al. 2001; Camerer et al. 2004; Costa-Gomes and Crawford 2006; Cai and Wang 2006; Crawford and Iriberri 2007). This kind of behavior type analysis is typically used to predict initial responses of subjects who play a game in normal form. If eye-tracking is used, then in order to gain additional information about strategic reasoning. 13

In contrast, our design is focused on the strategic use of gaze. The strategy space of a sender in the associated normal form consists of all possible gaze variations in addition to choices. Hence, if we want to identify behavior types, we need to restrict attention to the most important gaze characteristics. We will assume here that first movers only choose gaze (as in the LiveGaze-ForcedChoice treatment), and we characterize a gazing strategy by the box that is emphasized with gaze. There are potentially many ways in which a box could be emphasized, but we focus on looking at one box most or last, which were good predictors of choices in our non-strategic gaze data.

There are various assumptions about the anchoring L0 types in the literature, which all have in common that L0 is a non-strategic type. Particularly relevant for our setting are Crawford (2003), Cai and Wang (2006), and Ellingsen and Östling (2010), who argue that in games with communication, L0 behavior should be the naive strategy of sending only truthful signals. Accordingly, we assume that an L0 type exhibits gaze as if gaze was not transmitted. Our assumption about L0 receivers is that they are insensitive to payoffs and ignore any meaning that gaze might have, but that they favor salient options as in Crawford and Iriberri (2007). While saliency of locations as in their setting may also play a role in our design (e.g. of

\footnotetext{
13 For example, Polonio et al. (2015) have used their eye-tracking data to classify players as cognitive hierarchy types and predict some choices. Devetag et al. (2016) find unsophisticated choices but sophisticated search patterns. They arrive at a type classification that however only carries over to similar games.
} 
the top boxes), here we focus solely on gaze. A box is salient if the opponent's gaze emphasizes this box.

Type L1 then best responds to L0. As a second mover, an L1 type tries to infer choices from non-strategic gaze, i.e. forms beliefs about the opponent's choice according to the most-looked or last-looked heuristic. In the two coordination games and hide \& seek, an L1 receiver chooses an emphasized box. In the discoordination game, an L1 receiver chooses a non-emphasized box. A L1 first mover who best responds to a L0 second mover emphasizes and chooses the same box in the coordination game and different ones in hide \& seek and discoordination. An L2 type will then best respond to L1 behavior, and so on. Table 8 summarizes the behavior corresponding to the different levels of sophistication.

It becomes clear that the coordination games are strategically simple: All types above L0 manage to coordinate with gaze. Note also that with free choice of a box, TL is never chosen in the focal coordination game by any type above L0. The presence of L0 types or noise would mean a slightly smaller success rate, with possible choices of TL. One can also see that hide \& seek is a more complex game in which strategic sophistication matters a lot. The cyclical nature of the best responses in this game is reflected in the way the cognitive types cycle through all kinds of behavior. First movers of type L1 and L2 both try to mislead by emphasizing one box and choosing another. L3 types are typically rare and cannot be distinguished from an L0 type. However, one of the subjects in our experiment actually revealed this kind of thinking by stating that "[I looked] at the chosen box because I assumed that the respective other partner would not expect this".

The discoordination game is of intermediate complexity: Emphasizing a box with gaze is interpreted as "I have chosen this box" for some types or as "You should choose this box" for others. Again, the top left box is not chosen by sender or receiver above type L0 in this game. When playing the focal discoordination game with gaze, the types on average do not do very well since they do not take into account that they may misunderstand the sender's signal. A sophisticated type who plays a best response to a population of players with different levels of strategic sophistications may therefore be best off by choosing the top left box.

Furthermore, the classification should also include an equilibrium type. As described in our initial hypotheses, we expect equilibrium types to try to signal what they choose in the common interest games and exhibit completely uninformative gaze in the hide \& seek game. Unfortunately, uninformative gaze can-in equilibrium-be any gaze pattern. Not looking at all is clearly an uninformative gazing strategy, and distributing gaze evenly among all four boxes can also be interpreted as such.

Since the predictions for the behavior of the different types depend on the specified L0 types and because there are different ways to emphasize a box, one should not overestimate the predictive power of our interpretation of the behavior types. In 


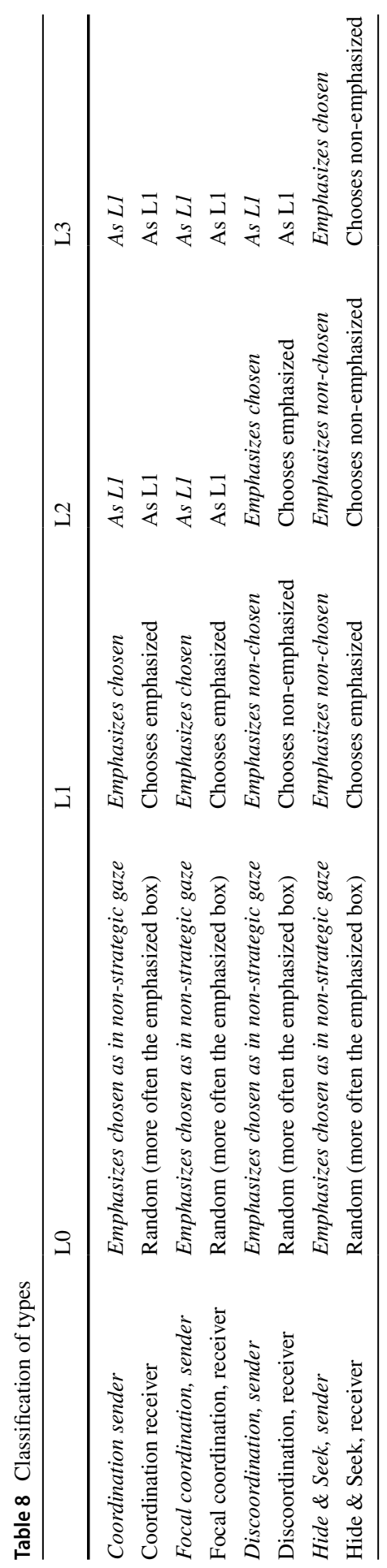


Table 9 Classification of first movers based on hide \& seek behavior

\begin{tabular}{|c|c|c|c|c|c|}
\hline \multirow{2}{*}{$\begin{array}{l}\text { Gaze style } \\
\text { Chosen option }\end{array}$} & \multicolumn{2}{|l|}{ Distributed gaze } & \multicolumn{2}{|c|}{ Looked mainly at one box } & \multirow[t]{2}{*}{ Mixing between } \\
\hline & Emphasized & Not emphasized & Emphasized & Not emphasized & \\
\hline NoGaze & $1(-)$ & $1(-)$ & $26(-)$ & $5(-)$ & $3(-)$ \\
\hline $\begin{array}{l}\text { LiveGaze- } \\
\quad \text { ForcedChoice }\end{array}$ & $1(0) ; \mathrm{D}(100 \%)$ & $6(0) ; \mathrm{D}(66.7 \%)$ & $6(3) ; \mathrm{D}(100 \%)$ & 14 (0); D (84.3\%) & $9(2) ; \mathrm{D}(62.2 \%)$ \\
\hline $\begin{array}{l}\text { LiveGaze- } \\
\quad \text { FreeChoice }\end{array}$ & $1(1) ; \mathrm{D}(100 \%)$ & 11 (3); D (61.8\%) & $5(2) ; \mathrm{D}(80 \%)$ & $10(1) ; \mathrm{D}(80 \%)$ & $9(1) ; \mathrm{D}(86.7 \%)$ \\
\hline
\end{tabular}

The table shows a classification of types based upon the gaze style (most looked at box received more than $40 \%$ of gaze) and features of the chosen option (emphasized or not). The remaining subjects are classified as mixing between rounds. The bracketed number shows how many subjects within a category behaved "weirdly" in the coordination and focal coordination games. The table also has the percentage of trials in which the categories chose an emphasized option in the focal discoordination game indicated by the percentage after the $\mathrm{D}$

the following, we explore whether we can categorize first movers in the hide \& seek game given their gaze and choice data (see Figs. 5, 6 in the Appendix for a graphical representation of the data).

We do a two-fold classification based on behavior in the hide \& seek game: 1) does any box have a larger share than $40 \%$ of gaze and 2) is the chosen box emphasized by either being looked at most or last. If 1) is true, then the strategy is counted as "looked mainly at one box", else as "distributed gaze". As each first mover made five choices, he qualifies to be a certain type if he plays the same strategy at least three times, which classifies 54 of the 72 subjects in the Live-treatments (see Table 9). ${ }^{14}$ The remaining subjects do not have one predominant strategy across the five rounds.

First, we see that there are very few people in NoGaze that distribute their gaze. This strategy, which is associated with an equilibrium type of the interactive treatments, is indeed used more often in the LiveGaze-treatments. Moreover, in the LiveGaze-treatments more subjects are in the category "choose the non-emphasized option" and thus try to actively mislead the second mover (corresponding to types L1 and L2 in Table 8).

We can further compare the types in the LiveGaze-treatments by considering whether they behave in a weird (L0 or random) manner in the coordination games: Either by spending less than $50 \%$ on the chosen box or by choosing a 10-point box in LiveGaze-FreeChoice, or by not choosing the required option in

\footnotetext{
${ }^{14}$ Requiring the same kind of strategy at least $80 \%$ of trials instead of $60 \%$ leads to having only 35 out of 72 subjects being assigned a strategic type.
} 
LiveGaze-ForcedChoice. As can be seen from the bracketed numbers in Table 9, subjects who mainly look at one box and choose an emphasized box in the hide \& seek game are more likely than the other categories to behave in a weird manner in the other coordination games.

Further, the level-k classification of Table 8 suggests different levels for emphasizing or non-emphasizing gaze of first movers in the discoordination game. Table 9 shows this information after the" $\mathrm{D}$ ": The number indicates the percentage of trials in which an emphasized option is chosen in the focal discoordination game within the categories. We see that subjects that choose a non-emphasized option in the hide \& seek game sometimes also choose the non-emphasized option (indicating an L1 type) and sometimes the emphasized option (indicating an L2 type) in the focal discoordination game. Subjects choosing an emphasized option in the hide \& seek game are also likely to choose the emphasized option in the focal discoordination game (indicating a non-strategic type).

\section{Discussion}

Our results show that people are generally able to correctly interpret non-strategic gaze in common interest games and, to a lesser extent, in a competitive hide \& seek game. The differences in the degree to which non-strategic gaze is understood can be explained by differences in the non-strategic gaze patterns: Subjects are more predictable when they choose the option that they look at last or for the longest time. Subjects strategically adapt their gaze when they are live transmitted to their current opponent, making their gaze particularly easy to interpret when their interests are perfectly aligned and making it more difficult to read when their interests are perfectly opposed.

We use simple games to explore the effects of gaze transfer, but the results also shed light on what can be expected in more complex situations. The strategic use of gaze is of importance in all situations in which people meet face-to-face and try to anticipate each other's actions, e.g. bargaining, interrogations, coordination, games or sports. The hide \& seek game, for instance, captures the strategic situation of penalty kicks in soccer. Indeed, Tay et al. (2010) find that goal keepers are worse at predicting the direction of a shot when the kicker is instructed to try to deceive the goalie, e.g. by looking in the opposite direction before taking the shot (Dicks et al. 2010; Nagano et al. 2006). Also, for other games, sports psychology has investigated how an athlete's action can be predicted from cues observable shortly before the action, often including the direction of gaze. Some studies also show that such cues can be mimicked in order to deceive the opponent [tennis (Rowe et al. 2009), rugby 
(Jackson et al. 2006), basketball (Sebanz and Shiffrar 2009), or handball (CañalBruland and Schmidt 2009)].

The general question how being observed changes behavior and how others will infer motives from subtle cues like the direction of gaze is likely to gain importance in the digital age, with implicit messages being read into response times and search patterns. With this broad interpretation, other considerations come into play, for example the role of experience and learning. We decided to not give any feedback and randomly re-match between rounds, but the question how learning via feedback affects strategic gaze is a natural continuation of this study. More research is needed to investigate whether strategically deceptive signals, be it with the eyes or via other cues, can systematically fool others. In particular, one would like to know whether people are overconfident regarding their ability to read cues or whether they learn to disregard cues that can easily be manipulated.

It would also be interesting to compare different kinds of signals. There is for example a recent literature that sheds light on the ability of people to predict what players will do from their facial expressions (Kovács-Bálint et al. 2013; van Leeuwen et al. 2018) or response times (Frydman and Krajbich 2017; Konovalov and Krajbich 2019). For cooperative situations, the question is whether it is easier to convey information using gaze, movement, facial expressions, language, or a combination. For competitive situations, it would be interesting to know whether some signals are more resistant to being mimicked than others.

Another open question is whether gaze can also signal things other than intentions such as sophistication, commitment, trustworthiness, attitude, or cooperativeness: Can people discern whether the gaze they see stems from an expert or novice? Are they able to predict how trustworthy or cooperative a person will act judging from her gaze? It would then again be interesting to see whether live gaze transmission is strategically utilized by the eye-tracked subjects also in these settings, for example to signal their own or a different type to evoke a specific response.

Acknowledgements Open Access funding provided by Projekt DEAL.

Open Access This article is licensed under a Creative Commons Attribution 4.0 International License, which permits use, sharing, adaptation, distribution and reproduction in any medium or format, as long as you give appropriate credit to the original author(s) and the source, provide a link to the Creative Commons licence, and indicate if changes were made. The images or other third party material in this article are included in the article's Creative Commons licence, unless indicated otherwise in a credit line to the material. If material is not included in the article's Creative Commons licence and your intended use is not permitted by statutory regulation or exceeds the permitted use, you will need to obtain permission directly from the copyright holder. To view a copy of this licence, visit http://creativecommons.org/licen ses/by/4.0/. 


\section{Appendix}

\section{Individual gaze and choice behavior}

The following two figures show a combination of both gaze and choice for all first movers in the Live-treatments for all five rounds of the hide \& seek game (Fig. 5 for LiveGaze-ForcedChoice and Fig. 6 for LiveGaze-FreeChoice). The x-axis distinguishes between the five rounds in the hide \& seek game, the y-axis shows the time share of the total time spent looking at the boxes for each box. The time share spent looking at a box is visualized using different colors for the respective boxes. After these four columns, there is always a black dot and a red cross. The black dot represents the time share spent on the chosen box, while the red cross represents the time share spent on the last looked at box, e.g. subject 301 looked at the TR box most and also last, but chose the BR box. Two subjects never looked at any box in all four rounds, e.g. subjects 336 or 410 , but do have data for the coordination games.

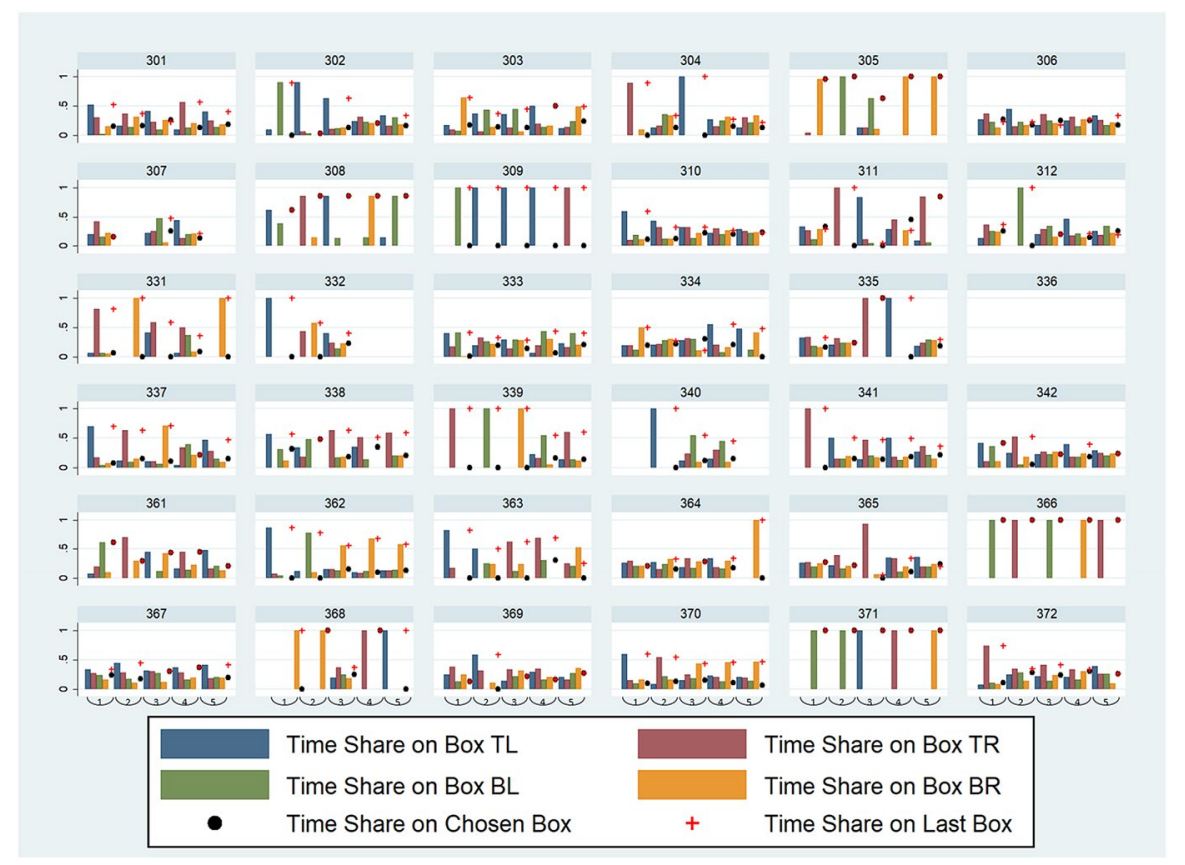

Fig. 5 Individual data of all subjects in the LiveGaze-ForcedChoice treatment. The x-axis shows the 5 periods within the hide \& seek game, while the y-axis shows the time share of total time for each box 


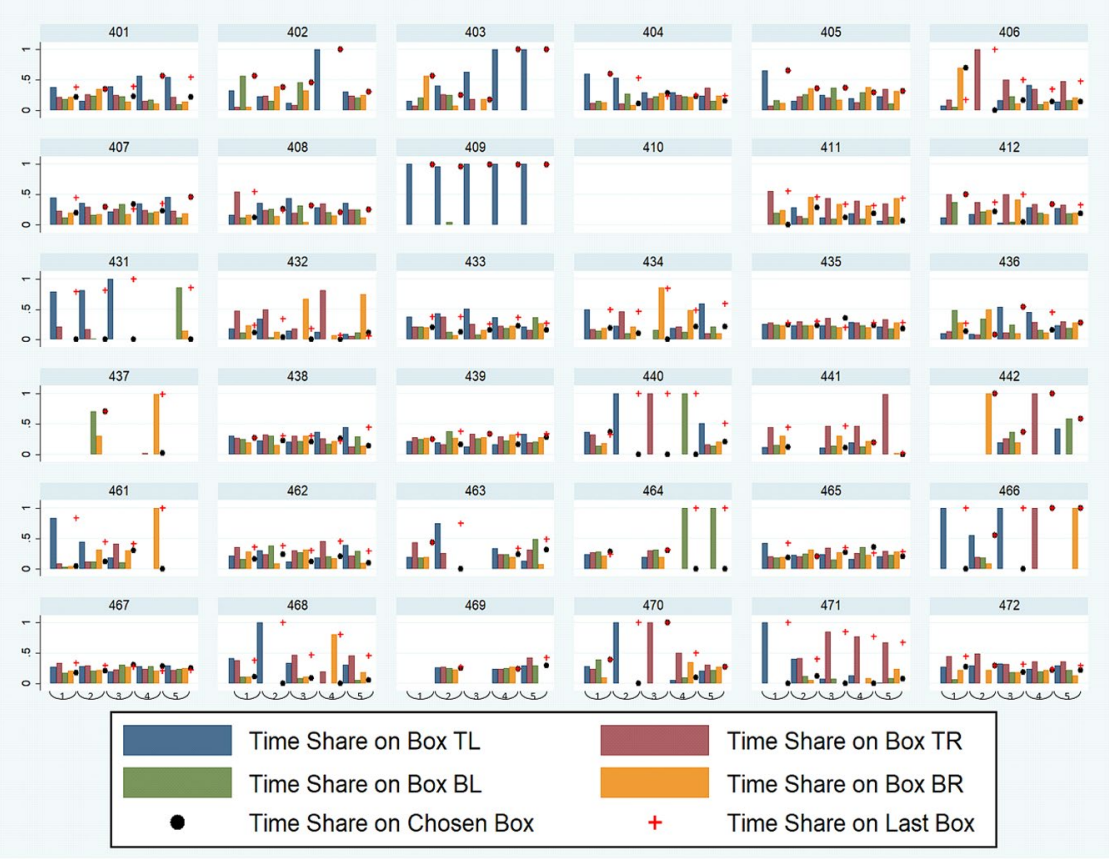

Fig. 6 Individual data of all subjects in the LiveGaze-FreeChoice treatment. The $\mathrm{x}$-axis shows the 5 periods within the hide \& seek game, while the y-axis shows the time share of total time for each box

\section{References}

Abed, F. (1991). Cultural influences on visual scanning patterns. Journal of Cross-Cultural Psychology, 22(4), 525-534.

Bardsley, N., Mehta, J., Starmer, C., \& Sugden, R. (2010). Explaining focal points: Cognitive hierarchy theory versus team reasoning. The Economic Journal, 120(543), 40-79.

Brennan, S. E., Chen, X., Dickinson, C. A., Neider, M. B., \& Zelinsky, G. J. (2008). Coordinating cognition: The costs and benefits of shared gaze during collaborative search. Cognition, 106(3), 1465-1477.

Brocas, I., Carrillo, J. D., Wang, S. W., \& Camerer, C. F. (2014). Imperfect choice or imperfect attention? Understanding strategic thinking in private information games. Review of Economic Studies, 81(3), 944-970.

Cai, H., \& Wang, J. T.-Y. (2006). Overcommunication in strategic information transmission games. Games and Economic Behavior, 56(1), 7-36.

Camerer, C. F., Ho, T.-H., \& Chong, J.-K. (2004). A cognitive hierarchy model of games. The Quarterly Journal of Economics, 119(3), 861-898.

Cañal-Bruland, R., \& Schmidt, M. (2009). Response bias in judging deceptive movements. Acta Psychologica, 130(3), 235-240.

Costa-Gomes, M., Crawford, V. P., \& Broseta, B. (2001). Cognition and behavior in normal-form games: An experimental study. Econometrica, 69(5), 1193-1235.

Costa-Gomes, M. A., \& Crawford, V. P. (2006). Cognition and behavior in two-person guessing games: An experimental study. American Economic Review, 96(5), 1737-1768.

Crawford, V. P. (2003). Lying for strategic advantage: Rational and boundedly rational misrepresentation of intentions. American Economic Review, 93(1), 133-149. 
Crawford, V. P., \& Iriberri, N. (2007). Fatal attraction: Salience, naivete, and sophistication in experimental "hide-and-seek" games. American Economic Review, 97(5), 1731-1750.

Devetag, G., Di Guida, S., \& Polonio, L. (2016). An eye-tracking study of feature-based choice in one-shot games. Experimental Economics, 19(1), 177-201.

Dicks, M., Button, C., \& Davids, K. (2010). Availability of advance visual information constrains association-football goalkeeping performance during penalty kicks. Perception, 39(8), 1111-1124.

Duchowski, A. T. (2007). Eye tracking methodology. Theory and Practice, 328(614), 2-3.

Ellingsen, T., \& Östling, R. (2010). When does communication improve coordination? American Economic Review, 100(4), 1695-1724.

Farrell, J. (1993). Meaning and credibility in cheap-talk games. Games and Economic Behavior, 5(4), 514-531.

Farrell, J., \& Rabin, M. (1996). Cheap talk. Journal of Economic Perspectives, 10(3), 103-118.

Fischbacher, U. (2007). z-Tree: Zurich toolbox for ready-made economic experiments. Experimental Economics, $10(2), 171-178$.

Foulsham, T., \& Lock, M. (2015). How the eyes tell lies: Social gaze during a preference task. Cognitive Science, 39(7), 1704-1726.

Frydman, C., \& Krajbich, I. (2017). Using response times to infer others' beliefs: An application to information cascades. Mimeo.

Gallagher-Mitchell, T., Simms, V., \& Litchfield, D. (2018). Learning from where 'eye'remotely look or point: Impact on number line estimation error in adults. The Quarterly Journal of Experimental Psychology, 71(7), 1526-1534.

Gibaldi, A., Vanegas, M., Bex, P. J., \& Maiello, G. (2017). Evaluation of the Tobii EyeX Eye tracking controller and Matlab toolkit for research. Behavior Research Methods, 49(3), 923-946.

Glöckner, A., \& Herbold, A. K. (2011). An eye-tracking study on information processing in risky decisions: Evidence for compensatory strategies based on automatic processes. Journal of Behavioral Decision Making, 24(1), 71-98.

Greiner, B. (2015). Subject pool recruitment procedures: Organizing experiments with ORSEE. Journal of the Economic Science Association, 1(1), 114-125.

Ho, T.-H., Camerer, C., \& Weigelt, K. (1998). Iterated dominance and iterated best response in experimental "p-beauty contests". The American Economic Review, 88(4), 947-969.

Holmes, K. J., Ayzenberg, V., \& Lourenco, S. F. (2016). Gamble on gaze: Eye movements reflect the numerical value of blackjack hands. Psychonomic Bulletin \& Review, 23(6), 1974-1981.

Holmqvist, K., Nyström, M., Andersson, R., Dewhurst, R., Jarodzka, H., \& Van de Weijer, J. (2011). Eye tracking: A comprehensive guide to methods and measures. Oxford: OUP.

Jackson, R. C., Warren, S., \& Abernethy, B. (2006). Anticipation skill and susceptibility to deceptive movement. Acta Psychologica, 123(3), 355-371.

Knoepfle, D. T., Wang, J. T.-Y., \& Camerer, C. F. (2009). Studying learning in games using eye-tracking. Journal of the European Economic Association, 7(2-3), 388-398.

Konovalov, A., \& Krajbich, I. (2019). Revealed strength of preference: Inference from response times. Judgment \& Decision Making, 14(4), 384-3944.

Kovács-Bálint, Z., Bereczkei, T., \& Hernádi, I. (2013). The telltale face: Possible mechanisms behind defector and cooperator recognition revealed by emotional facial expression metrics. British Journal of Psychology, 104(4), 563-576.

Krajbich, I., Armel, C., \& Rangel, A. (2010). Visual fixations and the computation and comparison of value in simple choice. Nature Neuroscience, 13(10), 1292.

Litchfield, D., \& Ball, L. J. (2011). Using another's gaze as an explicit aid to insight problem solving. Quarterly Journal of Experimental Psychology, 64(4), 649-656.

Litchfield, D., Ball, L. J., Donovan, T., Manning, D. J., \& Crawford, T. (2010). Viewing another person's eye movements improves identification of pulmonary nodules in chest x-ray inspection. Journal of Experimental Psychology: Applied, 16(3), 251-262.

Liu, C. (2008). The dark forest (J. Martinsen, Trans.). New York: Tor (translated version, 2015).

Müller, R., Helmert, J. R., \& Pannasch, S. (2014). Limitations of gaze transfer: Without visual context, eye movements do not help to coordinate joint action, whereas mouse movements do. Acta Psychologica, 152, 19-28.

Müller, R., Helmert, J. R., Pannasch, S., \& Velichkovsky, B. M. (2013). Gaze transfer in remote cooperation: Is it always helpful to see what your partner is attending to? Quarterly Journal of Experimental Psychology, 66(7), 1302-1316. 
Nagano, T., Kato, T., \& Fukuda, T. (2006). Visual behaviors of soccer players while kicking with the inside of the foot. Perceptual and Motor Skills, 102(1), 147-156.

Nagel, R. (1995). Unraveling in guessing games: An experimental study. The American Economic Review, 85(5), 1313-1326.

Nalanagula, D., Greenstein, J. S., \& Gramopadhye, A. K. (2006). Evaluation of the effect of feedforward training displays of search strategy on visual search performance. International Journal of Industrial Ergonomics, 36(4), 289-300.

Neider, M. B., Chen, X., Dickinson, C. A., Brennan, S. E., \& Zelinsky, G. J. (2010). Coordinating spatial referencing using shared gaze. Psychonomic Bulletin \& Review, 17(5), 718-724.

Polonio, L., Di Guida, S., \& Coricelli, G. (2015). Strategic sophistication and attention in games: An eyetracking study. Games and Economic Behavior, 94, 80-96.

Rowe, R., Horswill, M. S., Kronvall-Parkinson, M., Poulter, D. R., \& McKenna, F. P. (2009). The effect of disguise on novice and expert tennis players' anticipation ability. Journal of Applied Sport Psychology, 21(2), 178-185.

Rubinstein, A., Tversky, A., \& Heller, D. (1997). Naive strategies in competitive games. In W. Albers, W. Güth, P. Hammerstein, B. Moldovanu, \& E. van Damme (Eds.), Understanding strategic interaction: Essays in honor of Reinhard Selten (pp. 394-402). Berlin: Springer.

Salvucci, D. D., \& Goldberg, J. H. (2000). Identifying fixations and saccades in eye-tracking protocols. In Proceedings of the 2000 symposium on eye tracking research \& applications (pp. 71-78).

Schelling, T. C. (1960). The strategy of conflict. Cambridge, MA; London, England: Harvard University Press (reprinted and edited version, 1980).

Schwedes, C., \& Wentura, D. (2012). The revealing glance: Eye gaze behavior to concealed information. Memory \& Cognition, 40(4), 642-651.

Sebanz, N., \& Shiffrar, M. (2009). Detecting deception in a bluffing body: The role of expertise. Psychonomic Bulletin \& Review, 16(1), 170-175.

Shimojo, S., Simion, C., Shimojo, E., \& Scheier, C. (2003). Gaze bias both reflects and influences preference. Nature Neuroscience, 6(12), 1317.

Stahl, D. O., \& Wilson, P. W. (1995). On players' models of other players: Theory and experimental evidence. Games and Economic Behavior, 10(1), 218-254.

Stein, R., \& Brennan, S. E. (2004). Another person's eye gaze as a cue in solving programming problems. In Proceedings of the 6th international conference on multimodal interface (pp. 1-7).

Stewart, N., Gächter, S., Noguchi, T., \& Mullett, T. L. (2016). Eye Movements in Strategic Choice. Journal of Behavioral Decision Making, 29(2-3), 137-156. https://doi.org/10.1002/bdm.1901.

Tay, C. S., Chow, J. Y., Koh, M., \& Button, C. (2010). The effectiveness of keeper-independent penalty kicks using fake visual cues from penalty takers. International Journal of Sport Psychology, 43(5), 403-419.

van Leeuwen, B., Noussair, C. N., Offerman, T., Suetens, S., van Veelen, M., \& van de Ven, J. (2018). Predictably angry-Facial cues provide a credible signal of destructive behavior. Management Science, 64(7), 3352-3364.

van Wermeskerken, M., Litchfield, D., \& van Gog, T. (2018). What am I Looking at? Interpreting dynamic and static gaze displays. Cognitive Science, 42(1), 220-252.

Velichkovsky, B. M. (1995). Communicating attention: Gaze position transfer in cooperative problem solving. Pragmatics Cognition, 3(2), 199-223.

Vrij, A. (2004). Why professionals fail to catch liars and how they can improve. Legal and Criminological Psychology, 9(2), 159-181.

Wang, J. T.-Y., Spezio, M., \& Camerer, C. F. (2010). Pinocchio's pupil: Using eyetracking and pupil dilation to understand truth telling and deception in sender-receiver games. American Economic Review, 100(3), 984-1007.

Publisher's Note Springer Nature remains neutral with regard to jurisdictional claims in published maps and institutional affiliations. 